\title{
Mechanical properties of porous methyl silsesquioxane and nanoclustering silica films using atomic force microscope
}

\author{
C. Gaire $\cdot$ Y. Ou $\cdot$ H. Arao $\cdot$ M. Egami $\cdot$ \\ A. Nakashima $\cdot$ R. C. Picu · G.-C. Wang • \\ T.-M. Lu
}

(C) Springer Science+Business Media, LLC 2008

\begin{abstract}
Mechanical properties of porous methyl silsesquioxane samples with dielectric constant 2.4 and 2.0 and a recently developed nanoclustering silica film samples with dielectric constants 2.3 and 2.0 were evaluated using an atomic force microscope based nanoindentation. It was found that the Young's modulus and the hardness decrease while the fracture toughness increases with a decrease in the dielectric constant in the same type of material. Moreover, the Young's modulus and the hardness of the nanoclustering silica films were observed to be at least twice and fracture toughness values $\sim 1.3-1.5$ higher than those for methyl silsesquioxane films with similar dielectric constants. The high resolution topographic imaging capability of atomic force microscope was shown to be particularly useful in the measurement of cracks generated by the ultra-low indentation loads, and the evaluation of the fracture toughness of the nanoscale volumes of materials.
\end{abstract}

Keywords Methyl silsesquioxane ·

Nanoclustering silica Atomic force microscope $\cdot$

Fracture toughness $\cdot$ Hardness $\cdot$ Young's modulus

C. Gaire $(\bowtie)$ · Y. Ou · G.-C. Wang · T.-M. Lu

Department of Physics, Applied Physics and Astronomy,

Rensselaer Polytechnic Institute, Troy, NY 12180-3590, USA

e-mail: gairec@rpi.edu

H. Arao · M. Egami · A. Nakashima

Catalysts and Chemicals Ind. Co., Ltd., 13-2, Kitaminato-Machi,

Wakamatsu-Ku, Kitakyushu-Shi 808-0027, Japan

R. C. Picu

Department of Mechanical, Aeronautical and Nuclear

Engineering, Rensselaer Polytechnic Institute, Troy,

NY 12180-3590, USA

\section{Introduction}

In the development of high speed ultra-large scale integration (ULSI) devices, the inherent parasitic resistancecapacitance coupling leads to serious problems like signal propagation delay, crosstalk noise, and high power dissipation. To mitigate these problems, a combination of low dielectric constant (low- $k$ ) insulator with lower resistivity metal, e.g. $\mathrm{Cu}$, is required to replace the conventional interconnect metallization system made of silica $\left(\mathrm{SiO}_{2}\right)$ and Al. According to the International Technology Roadmap for Semiconductors (ITRS), dielectrics with $k<2$ are required for the metallization interconnect system of the $45 \mathrm{~nm}$ ULSI technology node and beyond [1]. In search of low- $k$ materials, there are two general directions that have seen a revival of interest. The first is the use of organic polymer based material whose dielectric constant is intrinsically smaller (compared to 4.5 of silica). For example, benzocyclobutene (BCB) and methyl silsesquioxane (MSQ), whose dielectric constants are 2.65 and 2.80 , respectively, have been studied as alternatives to conventional silica $[2,3]$. The strategy behind the silicabased materials is to use the network made of much stronger and more rigid silica as a starting point and then to reduce the $k$ value by lowering the density through the incorporation of chemical substituents or voids into the film [2]. For example, in the silsesquioxanes, each $\mathrm{Si}$ atom is bonded to one terminating group such as hydrogen, methyl, or phenyl, which results in a nominal stoichiometry $\mathrm{SiO}_{1.5} \mathrm{R}_{0.5}$. Both the cross linking density and material density are reduced by these terminating groups. The reduction in $k$ from that of $\mathrm{SiO}_{2}$ is thought to be mainly due to the reduction in density. The second approach to lower the $k$ value into ultra-low range is to introduce porosity into the silica by taking advantage of the low $k$ value of air 
$(k=1)$. Because very few dense materials have a $k$ value less than 2.5, there has been much interest in fabricating porous materials in order to reach ultra-low- $k$ values $(<2.0)$. In addition to the porous silica, porous MSQ has also been extensively studied for the use as ultra-low- $k$ material.

The introduction of porosity in the material lowers its $k$-value; however it is difficult to control the size and distribution of pores because of the formation of large interconnected pores by the coalescence of smaller ones. This is accompanied by a dramatic deterioration of the mechanical strength of the film, which significantly limits the yield of chemical mechanical polishing in low $-k / C u$ process integration [4]. Controlling the size and the spatial distribution of pores in porous materials is considered to be one of the most promising approaches to solve serious problems such as poor mechanical properties [5]. Recently, a novel coating type porous film from nanoclustering silica (NCS) has been developed in order to improve the low mechanical strength of the porous silsesquioxane films [6]. In this work, the NCS films were developed without the use of templates and were shown to have higher Young's modulus in comparison to conventional MSQ films. Also, by positron annihilation lifetime spectroscopy technique the NCS films were shown to have a very uniform pore distribution with reduced pore interconnectivity [7], an effect attributed to a molecular diffusion-like pore formation mechanism [8]. Moreover, a recent theoretical study [9] has shown that the pore size and pore position distribution affects the film's mechanical properties significantly. The above rationales have led us to conduct a systematic experimental study of the mechanical properties of the MSQ and NCS films.

Nanoindentation is a widely used method for the characterization of mechanical properties of thin films. In addition to the conventional nanoindenters, the use of atomic force microscope (AFM) for nanoindentation has experienced an increasing popularity [10-16]. In AFM nanoindentation, the usual imaging tip is replaced by a very sharp diamond tip which is used for both indentation and the subsequent imaging of the residual impressions. Specifically the nanoscale imaging capability of AFM provides an effective platform for measuring the mechanical properties of the materials in the regime where optical measurement of residual impression and crack sizes is difficult. This allows the nanoindentation technique to be scaled down to testing nanoscale volumes of materials, i.e. closer to conditions in which these materials will be used. In this article, we report the results of AFM nanoindentation experiments on four different types of samples: two MSQ samples with dielectric constants 2.4 and 2.0 (denoted as MSQ 2.4 and MSQ 2.0, respectively) and two NCS samples with dielectric constants 2.3 and 2.0 (denoted as NCS 2.3 and NCS 2.0, respectively). We report the effect of increasing porosity (leading to a lower dielectric constant) on the mechanical properties (Young's modulus, hardness and fracture toughness) of given material, i.e. MSQ 2.4 is compared to MSQ 2.0, and NCS 2.3 is compared to NCS 2.0. Further, we compare the results between the two types of materials with similar dielectric constants, i.e. MSQ 2.4 compared to NCS 2.3 and MSQ 2.0 compared to NCS 2.0 .

\section{Experimental details}

\subsection{Materials and samples}

Porous spin-on films of MSQ 2.4 and MSQ 2.0 were obtained from Motorola Inc. (Motorola, USA) and were prepared by standard method of porogen addition and removal by UV-curing. The MSQ is a silsesquioxane based material and has a chemical composition of $\left(\mathrm{CH}_{3}-\mathrm{SiO}_{3 / 2}\right)$. The NCS 2.3 and NCS 2.0 were obtained from CCIC (Catalysis and Chemicals Industries Co. Ltd, Japan). NCS is a silica based material with $k \leq 2.3$. Unlike MSQ, NCS is constitutively porous with a controlled distribution of pore size and position. The details of NCS sample preparation and properties are described in Ref. [6]. A summary of various properties (dielectric constant, film thickness, surface roughness, average pore size and porosity) of the samples used in the present work is given in Table 1. The dielectric constants and the film thicknesses were measured by capacitance measurement technique and ellipsometry, respectively; while the average pore diameter and the porosity were measured by ellipso-porosimetry technique [17]. The surface roughness of the samples was determined from tapping mode AFM topographic images. It can be seen from Table 1 that the average pore size for MSQ samples is higher in comparison to NCS samples with similar level of porosities. Moreover, the root mean square (rms) surface roughness of the MSQ samples is higher than their NCS counterparts. This indicates that NCS contains a higher number density of pores with smaller average wallto-wall distance between pores.

\subsection{Nanoindentation test}

Nanoindentation tests were carried out using an AFM (PSIA Inc.) in force-displacement mode to measure the Young's modulus, the hardness and the fracture toughness of the film samples. For the nanoindentation test, a stainless steel cantilever with a force constant of $271.9 \mathrm{~N} / \mathrm{m}$ and cube-corner diamond tip geometry was employed (Veeco, probe NICT-MTAP series). The force constant of the cantilever was calibrated by thermal calibration method 
Table 1 Summary of measured dielectric constant, surface roughness, film thickness and the vendor provided average pore size of the various samples used in the present experiments

\begin{tabular}{llllll}
\hline Film & Dielectric constant $k$ & Thickness $(\mathrm{nm})$ & Surface rms roughness $(\mathrm{nm})$ & Average pore size $(\mathrm{nm})$ & Porosity $p$ \\
\hline MSQ 2.0 & $2.0 \pm 0.1$ & $300 \pm 5$ & $1.32 \pm 0.05$ & $3.6 \pm 0.1$ & 0.49 \\
MSQ 2.4 & $2.4 \pm 0.1$ & $300 \pm 5$ & $0.95 \pm 0.03$ & $3.1 \pm 0.1$ & 0.33 \\
NCS 2.0 & $2.0 \pm 0.1$ & $150 \pm 5$ & $1.13 \pm 0.02$ & $2.1 \pm 0.1$ & 0.45 \\
NCS 2.3 & $2.3 \pm 0.1$ & $150 \pm 5$ & $0.70 \pm 0.01$ & $1.9 \pm 0.1$ & 0.33 \\
\hline
\end{tabular}

[18]. The Z-piezo detector was calibrated using standard calibration grating. The calibrated force constants and the calibrated Z-detector enable the conversion of the differential photodiode voltage (raw A-B signal) to force. This was done by pushing the tip of the cantilever (cantilever is attached to Z-piezo) a known distance against a sapphire surface while recording the raw A-B signal [19]. To conduct nanoindentation test, topographic image of the sample was taken in an area of interest (with the same tip used for indentation). The cantilever tip was brought in contact with the sample and pushed into the sample. The maximum force applied during the process was controlled by controlling the Z-displacement of the piezo. The force was measured from the cantilever deflection, while the specimen deformation was calculated by subtracting the cantilever deformation from the Z-displacement of the piezo. The samples were indented with maximum applied loads in the range of 5-60 $\mu \mathrm{N}$. A very slow loading rate $d Z / d t=10 \mathrm{~nm} / \mathrm{s}$ was employed to avoid the signature of material strain rate sensitivity (if any) in the data [16]. Each indent was made in a single load-unload sequence. After the indentation, the same tip was used to raster scan the specimen surface in tapping mode to take the topographic image of the residual impression on the sample. The indentation tests were conducted at 25 different points on each sample. The load versus the specimen deformation for each indentation was plotted and used for data analysis. All the measurements were carried out at room temperature $\left(\sim 25^{\circ} \mathrm{C}\right)$ and at a relative humidity of $\sim 50 \%$.

\subsection{Indenter characterization}

The indenter tip was used to image the AFM tip characterization gratings (silicon spikes with radius of curvature $<10 \mathrm{~nm}$; TGT01 series, MikroMasch, Chapel Hill, NC, USA) in tapping mode and the image was de-convoluted to obtain the image of the indenter tip. Figure 1a shows the de-convoluted image of the tip. The tip is slightly asymmetric with the highest asymmetry along the line marked 3 in Fig. 1a. Also, the height profile of the indenter tip along the line 2 in Fig. 1a is shown in Fig. 1b. This line profile along with other line profiles were used to determine the total included angle of the indenter tip along three directions. We found that, the three angles were slightly off from $90^{\circ}$ (included angle for ideal cube corner geometry), and were $89.4 \pm 0.2^{\circ}, 91.0 \pm 0.5^{\circ}$ and $97.5 \pm 0.5^{\circ}$ along lines 1,2 and 3, respectively. The tip's radius of curvature was determined to be $\sim 47 \mathrm{~nm}$. The distance denoted as $\delta$ in Fig. $1 \mathrm{~b}$ was determined to be $\sim 25 \mathrm{~nm}$. The corresponding area function for the indenter at a contact depth $h_{c}$ was calculated using the following expression $[20,21]$ :

$A_{c}=\beta\left(h_{c}+\delta\right)^{2}$.
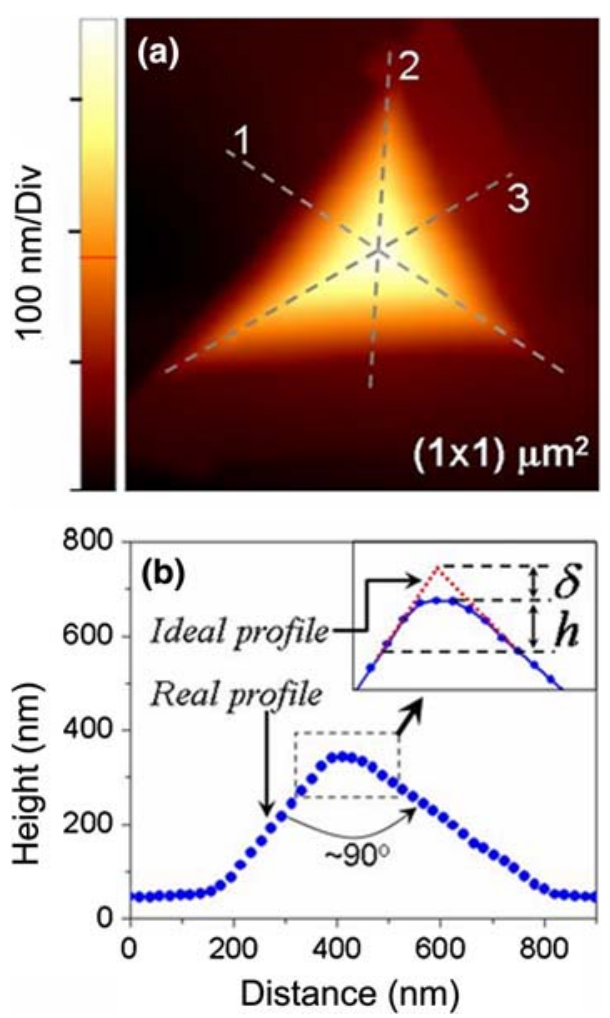

Fig. 1 Method used in the indenter area function characterization. a AFM image of the cube corner diamond indenter tip. The height profiles along lines 1,2 , and 3 were used to determine the total included angles of the tip and were found to be $89.4 \pm 0.2^{\circ}$, $91.0 \pm 0.5^{\circ}$ and $97.5 \pm 0.5^{\circ}$, respectively. b Line profile along line 2 of (a). The inset is the magnification of the rectangular enclosure in the line profile. The parameter $\delta$ is the distance from the blunt extremity of the tip to the point where the best fit lines of the sides meet. At a depth $h$ of the indenter the area function is given by $A(h)=\beta(h+\delta)^{2}$ where $\beta$ is a constant to be determined 
Here the coefficient $\beta$ for our indenter geometry was determined based on the three angles to be 3.03. Note that for an indenter with ideal cube corner geometry $(\delta=0)$, the expression for the area function reads as $A_{c}=$ $2.598 h_{c}^{2}$.

\section{Results and discussion}

\subsection{Young's modulus and hardness}

A typical tapping mode AFM image $\left(2 \times 2 \mu \mathrm{m}^{2}\right)$ of the MSQ 2.0 surface is shown in Fig. 2a. The circled numbers $1,2,3$, and 4 represent the regions chosen for indentation. Nanoindentation tests were carried out with maximum loads $14.8,21.5,29.0$ and $36.7 \mu \mathrm{N}$ on locations $1,2,3$, and 4 , respectively. Figure $2 \mathrm{~b}$ shows the tapping mode AFM image of the same surface in Fig. 2a right after indentation. These images are obtained using the same tip used for indentation. Note that the contrast scale in Fig. $2 b$ is 10 times larger than that in Fig. 2a. Line profiles across the indents in various directions were used to determine the residual depth, $h_{c}$. In Fig. 2 b, it is clearly seen that plastic deformation occurred in the films and the residual impressions are triangular with small cracks going outward (radial cracks) from the indenter corners. Figure $2 \mathrm{c}$ shows the load-displacement $(P-h)$ curves corresponding to indentations at locations $1,2,3$, and 4 in Fig. $2 b$. The curves exhibit the same loading path, with different residual strains upon unloading because of different maximum loads used. The slopes of the unloading curve at the maximum depths were used to calculate the Young's modulus.

A comparison of nanoindentation $P-h$ curves for MSQ and NCS films is shown in Fig. 3a, b. In Fig. 3a, we compared the $P-h$ curves for MSQ 2.4 and MSQ 2.0 films for approximately same maximum applied load, $P_{\max } \sim 11 \mu \mathrm{N}$. It can be seen that as the dielectric constant of the material is lowered (e.g. from $k=2.4$ to 2.0), the material becomes softer. Similarly, in Fig. $3 \mathrm{~b}$, the $P-h$ curves for NCS 2.3 and NCS 2.0 films are compared for approximately the same maximum applied load, $P_{\max } \sim 15 \mu \mathrm{N}$. These curves demonstrate a similar trend.

Next, we compare the $P-h$ curves obtained from different materials but having similar dielectric constants. For example, we compare the $P-h$ curve of MSQ 2.4 in Fig. 3a and that of NCS 2.3 in Fig. 3b. Even though the dielectric constants are similar, NCS is stiffer. A similar conclusion results by comparing MSQ 2.0 with NCS 2.0. This effect can be due to either or both the intrinsic stiffness of the two materials (notwithstanding the voids), and to a void size effect introduced by the fact that the average pore size in
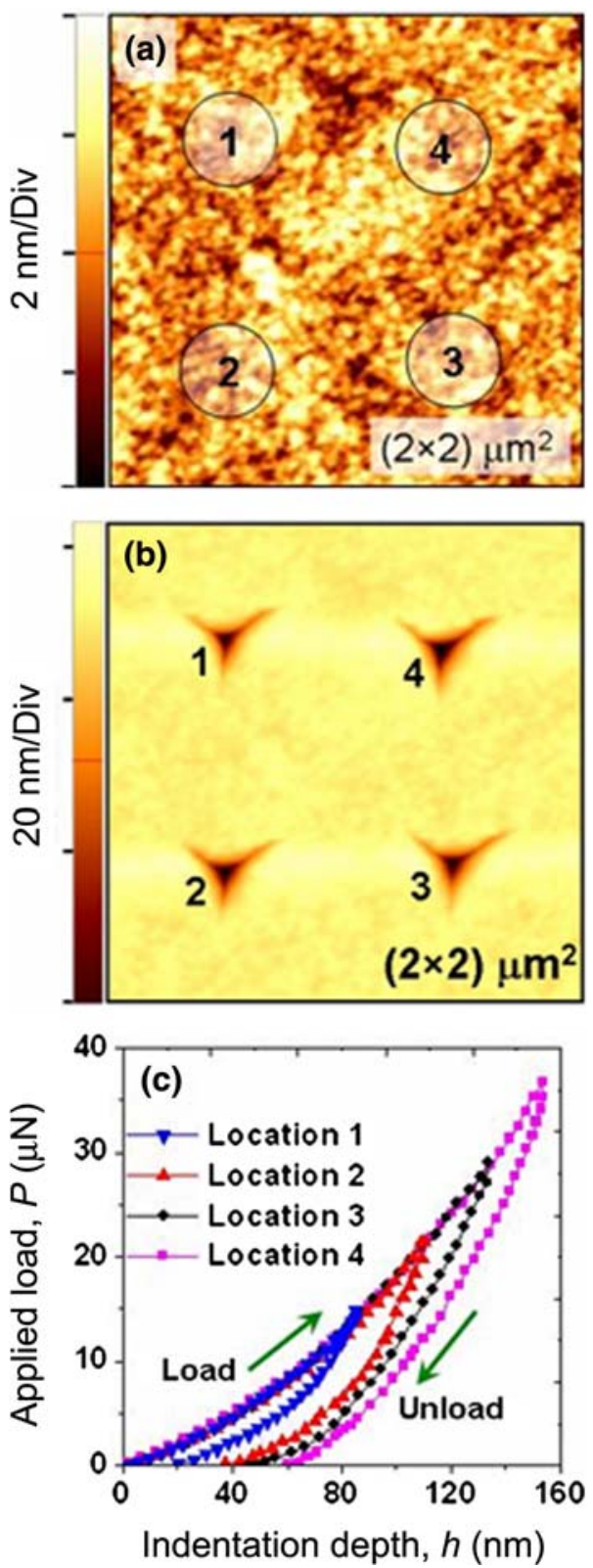

Fig. 2 a A tapping mode AFM image of the MSQ 2.0 surface taken by the indenter tip. The circled numbers $1,2,3$, and 4 are the points chosen for indentation. b A tapping mode AFM image of the same surface in (a) right after indentation and taken by the same tip used for indentation. c Load-displacement curves corresponding to the indentations at locations $1,2,3$, and 4 in (b). The maximum forces used were $14.8,21.5,29.0$ and $36.7 \mu \mathrm{N}$ on locations $1,2,3$, and 4 , respectively. The loading rate was kept low at $2.0 \mu \mathrm{N} / \mathrm{s}(d Z /$ $d t=10 \mathrm{~nm} / \mathrm{s}$ ) for all the indentations. The curves have the same loading path, with different residual depths upon unloading because of different maximum loads used. The slope of the unloading curve at maximum depth was used to calculate the contact depth, hardness and Young's modulus

NCS is smaller than in MSQ. A pore size effect on both moduli and yield stress was evidenced in other micro- and nanoporous materials; the yield/flow stress increases 

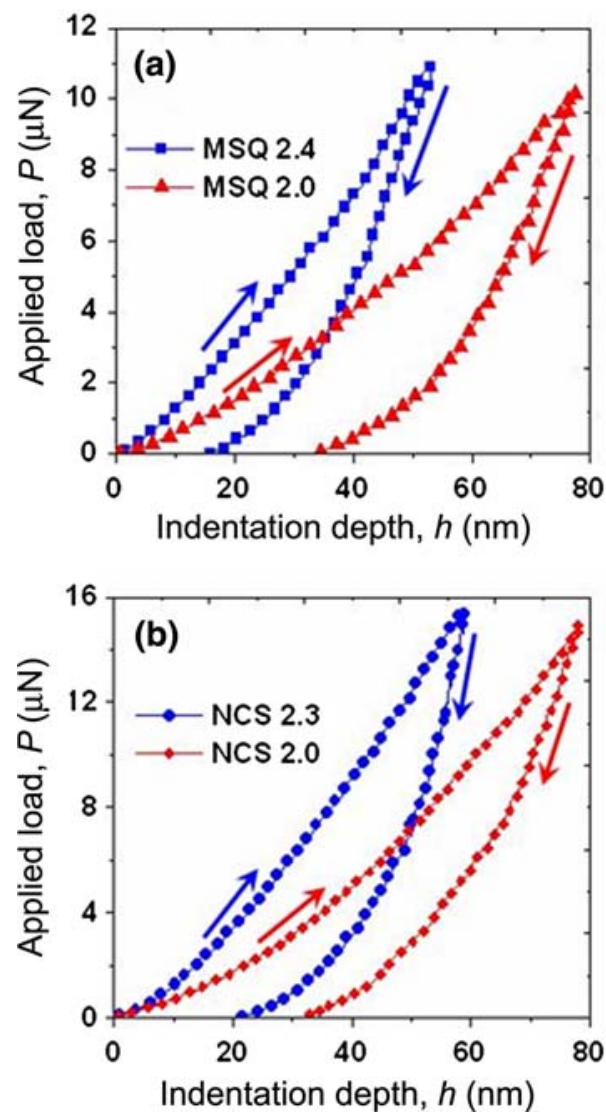

Fig. 3 Comparison of load-displacement $(P-h)$ curves during the nanoindentation of various films. Comparison of the curves for a MSQ 2.4 and MSQ 2.0 films; b NCS 2.3 and NCS 2.0 films. Maximum loads used in (a) and (b) were $\sim 11$ and $\sim 15 \mu \mathrm{N}$, respectively. Comparison of the unloading slope and the residual depths in the same species of materials indicates that the material with lower dielectric constant is in general softer. The comparisons of the $P-h$ curves for MSQ 2.4 in (a) and NCS 2.3 in (b) shows that more load has to be applied for NCS 2.3 in order to produce the same maximum indentation depths of $\sim 55 \mathrm{~nm}$. Likewise, a comparison of the $P-h$ curves for MSQ 2.0 in (a) and NCS 2.0 in (b) shows that more load has to be applied for NCS 2.3 to produce the same maximum indentation depth of $\sim 75 \mathrm{~nm}$

dramatically with decreasing the pore size at constant porosity [22]. The effect on stiffness depends on the surface tension of the respective bulk material and is still a matter of debate [23, 24]. Since non-porous NCS is not available for testing, we cannot draw a conclusion here regarding the relative importance of the two effects. However, we conclude that, at the same dielectric constant, NCS has better mechanical resistance.

The $P-h$ curves were utilized to calculate the hardness and Young's modulus of various films using the Oliver and Pharr method [20]. First of all, the unloading portion of the load-displacement curve was fitted using the power law:
$P=B\left(h-h_{f}\right)^{m}$

The slope of the curve at maximum load was found by differentiating Eq. 2 and evaluating it at $h=h_{\max }$.

$S=\left.\frac{d P}{d h}\right|_{h=h_{\max }}=m B\left(h-h_{f}\right)^{m-1}$

The contact depth $h_{c}$ was determined from

$h_{c}=h_{\max }-\varepsilon\left(\frac{P_{\max }}{S}\right)$

Here $\varepsilon$ depends on geometry of the indenter [25] and was taken to be 0.75 .

The hardness $(H)$ and the Young's modulus $\left(E_{s}\right)$ were calculated as:

$H=\frac{P_{\max }}{A_{c}}$

and

$E_{r}=\frac{\sqrt{\pi}}{2 \gamma} \frac{S}{\sqrt{A_{c}}} ; \frac{1}{E_{r}}=\frac{1-v_{s}^{2}}{E_{s}}+\frac{1-v_{i}^{2}}{E_{i}}$

The contact area, $A_{c}$, required in Eqs. 5 and 6 was evaluated from Eq. 1. The subscripts $s$ and $i$ represent the specimen and the indenter, $v$ represents the Poisson's ratio, and $\gamma$ is a numerical factor equal to 1.034 for cube corner geometry. For tests where the ratio $h_{\max } / t$ is high $(>30 \%)$ ( $t=$ film thickness), the hardness and modulus data are likely to be influenced by the presence of the substrate, so the substrate correction following the method proposed by Saha and Nix [26] combined with King's analysis [27] was employed for the Young's modulus data. However the hardness data was not corrected since it requires the knowledge of the yield stress to be input in the expression; this parameter could not be measured for these samples.

A summary of measured mechanical properties is listed in Table 2. Note that the uncertainties in the Young's modulus, hardness and fracture toughness given in Table 2 are the standard errors of the measured parameters. From Table 2, it is seen that the hardness and Young's modulus decrease as the dielectric constant is lowered in the same type of material due to increasing porosity. For example, the hardness and the Young's modulus of MSQ 2.0 are $\sim 1.6$ and 1.2 times lower, respectively than those of MSQ 2.4. Similarly, the hardness and the Young's modulus of NCS 2.0 are 2.3 and 2.0 times lower, respectively than those of NCS 2.3. Comparing NCS 2.0 and MSQ 2.0, both having the same dielectric constant, our results show that NCS has higher modulus and hardness. A similar conclusion is obtained when comparing NCS 2.3 and MSQ 2.4. This makes NCS more suitable for microelectronic applications than MSQ. 
Table 2 Summary of measured maximum applied load $\left(P_{\max }\right)$, ratio of maximum indentation depth to the film thickness $\left(h_{\max } / t\right)$, hardness $(H)$, Young's modulus $(E)$ and fracture toughness $(K)$ of various films studied

\begin{tabular}{llllll}
\hline Film & Applied load $P_{\max }(\mu \mathrm{N})$ & Range of $h_{\max } / t$ & Hardness $H(\mathrm{GPa})$ & Young's modulus $E(\mathrm{GPa})$ & Toughness $K(\mathrm{MPa} \sqrt{\mathrm{m}})$ \\
\hline MSQ 2.0 & $5-38$ & $0.25-0.55$ & $0.15 \pm 0.02$ & $1.94 \pm 0.05$ & $0.042 \pm 0.002$ \\
MSQ 2.4 & $5-42$ & $0.18-0.43$ & $0.24 \pm 0.02$ & $2.31 \pm 0.12$ & $0.032 \pm 0.003$ \\
NCS 2.0 & $5-45$ & $0.50-0.85$ & $0.30 \pm 0.04$ & $3.45 \pm 0.06$ & $0.061 \pm 0.005$ \\
NCS 2.3 & $5-50$ & $0.34-0.65$ & $0.68 \pm 0.03$ & $6.82 \pm 0.35$ & $0.043 \pm 0.003$ \\
\hline
\end{tabular}

The average Young's modulus and hardness for nonporous MSQ $(k=2.80)$ are reported as 4.41 and $0.28 \mathrm{GPa}$, respectively [28]. As expected, making the material porous reduces the modulus to 2.31 and $1.94 \mathrm{GPa}$ for porosities $p=0.33(k=2.4)$ and $p=0.49(k=2.0)$, respectively. In the same samples, the hardness decreases from $0.28 \mathrm{GPa}$ to 0.24 and $0.15 \mathrm{GPa}$, respectively. Not surprisingly, a similar trend in the values obtained by conventional nanoindentation is observed in other materials. For example, porous amorphous silica with dielectric constant $k=2$ has Young's modulus $3.33 \mathrm{GPa}$ and hardness $\sim 0.38 \mathrm{GPa}$, while in a more dense state (with dielectric constant $k=2.3$ ), has higher modulus $(5.49 \mathrm{GPa})$ and hardness (0.7 GPa) [29-31].

\subsection{Fracture toughness}

The AFM-based nanoindentation technique was further utilized to evaluate the fracture toughness of the MSQ and NCS films. A schematic of the indentation-induced cracks is shown in Fig. 4a, where $c$ represents the crack length and $a$ represents the impression size. The condition for a well developed crack is cited [32, 33] as $c / a>2$. Based on the fracture mechanics analysis, the fracture toughness, $K_{I C}$, and the crack length, $c$, are related as [34]

$K_{I C}=B\left(\frac{E}{H}\right)^{1 / 2}\left[\frac{P}{c^{3 / 2}}\right]$

where, $B$ is an empirical constant that depends on the indenter geometry, $E$ is the Young's modulus, $H$ is the hardness and $P$ is the maximum applied load leading to cracks of length $c$. For cube corner indenter geometry, cracks produced are dominantly radial and the constant $B$ is equal to 0.032 [35]. By using Eq. 7, it is apparent that the evaluation of fracture toughness not only requires the knowledge of the modulus and hardness but also a precise measurement of the crack size for a given indentation load. In micro-indentation, cracks at relatively high indentation loads of several hundred grams are on the order of $100 \mu \mathrm{m}$ and can be measured optically. However, the measurement of fracture toughness of very thin films or small volumes of materials requires much smaller indentations. While the cracking threshold can be substantially reduced by using sharp indenters such as those with cube corner geometry $[35,36]$, the measurement of extremely small crack sizes by optical methods is virtually impossible. With the high resolution topographic imaging capability of the AFM, it is possible to precisely measure indentation impression size and the length of the cracks. For example in Fig. 4b, the AFM topographic image of the indented surface is shown. The dotted triangle shown in Fig. $4 \mathrm{~b}$ is the hardness impression. Cracks are seen to propagate in the radial direction starting from the vertices of the dotted triangle. To precisely measure the size of the cracks, the contrast in the topographic image (Fig. 4b) was further enhanced by using a high pass filter. Figure $4 \mathrm{c}$ shows the image in Fig. $4 \mathrm{~b}$ after high pass filtering. In this way, the crack sizes were readily measurable and used in the Eq. 7 along with the values of hardness and modulus to calculate the fracture toughness of the samples.

A summary of the calculated fracture toughness values of various samples studied is given in Table 2. The fracture toughness of MSQ 2.0 is $\sim 1.3$ times higher than that of MSQ 2.4. Similarly, the fracture toughness of NCS 2.0 is 1.4 times higher than that for NCS 2.3. This indicates that toughness is strongly influenced by porosity. Porosity increases the toughness of microporous materials due to several effects: crack trapping, void growth/cavitation in the process region in front of the crack tip, perturbation of local stress field. In nanoporous intrinsically brittle materials, the void growth argument does not hold, but some degree of crack trapping is expected. An additional effect that may play a role is the fact that increasing porosity decreases the moduli which reduces the driving force for crack propagation at given displacement-imposed boundary conditions.

Comparing NCS 2.0 and MSQ 2.0, both having the same dielectric constant and porosity, indicates that NCS has higher fracture toughness. A similar conclusion is obtained when comparing NCS 2.3 and MSQ 2.4. This indicates a pore size effect. As the pore dimensions decrease at constant porosity, the average pore wall-to-wall distance decreases and pore-pore interaction is more pronounced. A related effect was observed by Xia et al. [37] while testing nanoporous anodic alumina membranes. They used a similar technique and determined that when the pores are small enough, buckling instabilities of the pore walls 


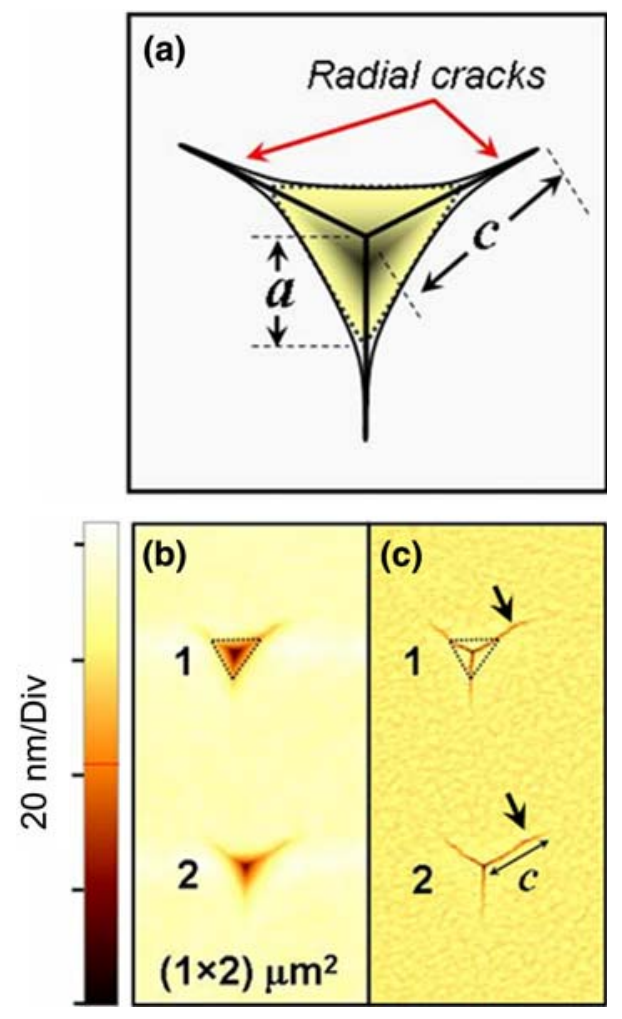

Fig. 4 a A schematic showing the hardness impression size, $a$, and the crack length, $c$. b A $\left(1 \times 2 \mu \mathrm{m}^{2}\right)$ tapping mode AFM topographic image of the surface right after indentation. The dotted triangle shows the hardness impression due to the cube corner geometry indenter, and the dark lines going outward from the vertices of this triangle are the cracks. c A high pass filtering of image shown in (b)

leading to shear banding starting from indenter edges are observed rather than cracks. This implies higher toughness for samples with smaller voids and given porosity. Since our samples do not have the open pore structure of their alumina templates (with cylindrical pores running perpendicular to the film surface), we do not observe such instabilities, but do not exclude that a similar effect may underlie the observed increased toughness.

In closure it should be noted that an error in the fracture toughness measurement may be introduced by the fact that for $(c / a)$ in the vicinity of 2, plastic deformation under the indenter may affect crack propagation [33]. Other considerations specific to cube-corner indenters [38] may also be important. Considering all these issues, we estimate the uncertainty of the reported fracture toughness values to be $\sim 40 \%$ [39].

\section{Conclusion}

An AFM was used to evaluate the Young's modulus, the hardness and the fracture toughness of porous MSQ and
NCS films. It was demonstrated that an increase in the porosity of the film (or a decrease in the dielectric constant) decreases the Young's modulus and the hardness of the film, while increasing the fracture toughness. It was also shown that the NCS films have better mechanical properties than MSQ samples and carry a promising potential usage in the interconnect industries.

Acknowledgements This work was partially supported by NSF under Grant No. 0324492. Any opinions, findings and conclusions or recommendations expressed in this material are those of the authors and do not necessarily reflect the views of the NSF. We thank G. Spencer from Freescale Motorola and Sammy Saito from CCIC for providing MSQ and NCS samples, respectively.

\section{References}

1. S. Amakawa et al., in Interconnect (International Technology Roadmap for Semiconductors, Semiconductor Industry Association, San Jose, CA, 2007), p. 7

2. K. Maex, M.R. Baklanov, D. Shamiryan, F. Iacopi, S.H. Brongersma, Z.S. Yanovitskaya, J. Appl. Phys. 93, 8793 (2003). doi: $10.1063 / 1.1567460$

3. M. Morgen, E.T. Ryan, J.-H. Zhao, C. Hu, T. Cho, P.S. Ho, Annu. Rev. Mater. Sci. 30, 645 (2000). doi:10.1146/annurev. matsci.30.1.645

4. X. Xiao, N. Hata, K. Yamada, T. Kikkawa, Rev. Sci. Instrum. 74, 4539 (2003). doi:10.1063/1.1611615

5. A. Grill, Y. Iba, H. Kitada, N. Nishikawa, M. Miyajima, S. Fukuyama et al., in Advanced Metallization Conference (2001), p. 253

6. T. Nakamura, A. Nakashima, in International Interconnect Technology Conference (Institute of Electrical and Electronic Engineers, San Francisco, CA, 2004), p. 175

7. K. Ito, R.-S. Yu, K. Sato, K. Hirata, Y. Kobayashi, T. Kurihara et al., J. Appl. Phys. 98, 094307 (2005). doi:10.1063/1.2125121

8. M. Ikeda, J. Nakahira, Y. Iba, H. Kitada, N. Nishikawa, M. Miyajima et al., in International Interconnect Technology Conference (Institute of Electrical and Electronic Engineers, San Fransisco, CA, 2003), p. 71

9. H. Miyoshi, N. Hata, T. Kikkawa, Jpn. J. Appl. Phys. 44, 1166 (2005). doi:10.1143/JJAP.44.1166

10. S. Garcia-Manyes, A.G. Güell, P. Gorostiza, F. Sanza, J. Chem. Phys. 123, 114711 (2005). doi:10.1063/1.2035094

11. A.V. Kulkarni, B. Bhushan, Thin Solid Films 290-291, 206 (1996). doi:10.1016/S0040-6090(96)08974-2

12. M. Petzold, J. Landgraf, M. Futing, J.M. Olaf, Thin Solid Films 264, 153 (1995). doi:10.1016/0040-6090(95)05855-9

13. S. Chowdhury, M.T. Laugier, Nanotechnology 15, 1017 (2004). doi:10.1088/0957-4484/15/8/027

14. M.R. VanLandingham, T.F. Juliano, M.J. Hagon, Meas. Sci. Technol. 16, 2173 (2005). doi:10.1088/0957-0233/16/11/007

15. J. Domke, M. Radmacher, Langmuir 14, 3320 (1998). doi: 10.1021/1a9713006

16. D.-L. Liu, T.-M. Lu, G.-C. Wang, R.C. Picu, Appl. Phys. Lett. 85, 3053 (2004). doi:10.1063/1.1805710

17. M.R. Baklanov, K.P. Mogilnikov, V.G. Polovinkin, F.N. Dultsev, J. Vac. Sci. Technol. B 18, 1385 (2000). doi:10.1116/1.591390

18. J.L. Hutter, J. Bechhoefer, Rev. Sci. Instrum. 64, 1868 (1993). doi:10.1063/1.1143970

19. C. Gaire, D.-X. Ye, F. Tang, R.C. Picu, G.-C. Wang, T.-M. Lu, J. Nanosci. Nanotech. 5, 1893 (2005)

20. W.C. Oliver, G.M. Pharr, J. Mater. Res. 7, 1564 (1992). doi:10.1557/JMR.1992.1564 
21. M. Martin, T. Troyon, J. Mater. Res. 17, 2227 (2002). doi: 10.1557/JMR.2002.0328

22. M. Hakamada, M. Mabuchi, Scr. Mater. 56, 1003 (2007). doi: 10.1016/j.scriptamat.2007.01.046

23. R.E. Miller, V.B. Shenoy, Nanotechnology 11, 139 (2000). doi: 10.1088/0957-4484/11/3/301

24. L.H. He, Z.R. Li, Int. J. Solids Struct. 43, 6208 (2006). doi: 10.1016/j.ijsolstr.2005.05.041

25. W.C. Oliver, G.M. Pharr, J. Mater. Res. 19, 3 (2004). doi: 10.1557/jmr.2004.19.1.3

26. R. Saha, W.D. Nix, Acta Mater. 50, 23 (2002). doi: 10.1016/S1359-6454(01)00328-7

27. R.B. King, Int. J. Solids Struct. 23, 1657 (1987). doi: 10.1016/0020-7683(87)90116-8

28. A.M. Padovani, L. Rhodes, L. Riester, G. Lohman, B. Tsuie, J. Conner et al., Electrochem. Solid-State Lett. 4, F25 (2001). doi: $10.1149 / 1.1403215$

29. G. Xu, J. He, E. Andideh, J. Bielefeld, T. Scherban, in IEEE International Interconnect Technology Conference (Piscataway, NJ, San Francisco, CA, 2002), p. 57

30. X. Huang, A.A. Pelegri, J. Eng. Mater. Technol. 125, 361 (2003). doi:10.1115/1.1605109
31. Z. Li, M.C. Johnson, M. Sun, E.T. Ryan, D.J. Earl, W. Maichen et al., Angew. Chem. Int. Ed. 45, 6329 (2006). doi: 10.1002/anie. 200602036

32. D.B. Marshall, B.R. Lawn, J. Am. Ceram. Soc. 60, 87 (1977). doi:10.1111/j.1151-2916.1977.tb16106.x

33. R. Tandon, T.E. Buchheit, J. Am. Ceram. Soc. 90, 502 (2007). doi:10.1111/j.1551-2916.2006.01389.x

34. B.R. Lawn, A.G. Evans, D.B. Marshall, J. Am. Ceram. Soc. 63, 573 (1980)

35. G.M. Pharr, D.S. Harding, W.C. Oliver, in Mechanical Properties and Deformation Behavior of Materials Having Ultra-Fine Microstructures, ed. by M. Natasi, D.M. Parkin, H. Gleiter (Kluwer Academic, Dordrecht, Netherlands, 1993), p. 449

36. X. Li, B. Bhushan, Thin Solid Films 315, 214 (1998). doi: 10.1016/S0040-6090(97)00788-8

37. Z. Xia, L. Riester, B.W. Sheldon, W.A. Curtin, J. Liang, A. Yin et al., Rev. Adv. Mater. Sci. 6, 131 (2004)

38. D.J. Morris, A.M. Vodnick, R.F. Cook, Int. J. Fract. 136, 265 (2005). doi:10.1007/s10704-005-6033-x

39. G.M. Pharr, Mater. Sci. Eng. A 253, 151 (1998). doi:10.1016/ S0921-5093(98)00724-2 